\title{
UM OBJETO DE APRENDIZAGEM PARA O ESTUDO INTERATIVO DA GLICOGÊNESE HEPÁTICA PELAS VIAS DIRETA E INDIRETA
}

\author{
Vera M. T. Trindade ${ }^{1}$, Bruno S. Pastoriza ${ }^{1,2}$, Luciene P. Vianna ${ }^{1}$, Christianne G. \\ Salbego ${ }^{1}$ e José C. Del Pino ${ }^{1,2}$ \\ ${ }^{I}$ Grupo de Criação de Objetos Educacionais em Bioquímica-Dep. Bioquímica-ICBS-UFRGS, ${ }^{2}$ Área de Educação \\ Química-IQ-UFRGS, Porto Alegre-RS-Brasil.
}

\section{Resumo:}

As disciplinas de Bioquímica básica, normalmente, utilizam aulas expositivas ou a dinâmica de estudo em grupo como estratégias de ensino teórico. A revisão dos conceitos abordados em sala de aula é realizada, frequentemente, através de exercícios clássicos de fixação. Por outro lado, nos últimos anos, os livros texto têm sido acompanhados de CDs, que contem várias de suas figuras animadas e vinculadas a hipertextos e a links de páginas da internet, que facilitam a construção de significados do conhecimento bioquímico. Paralelamente, objetos de aprendizagem em ambientes virtuais têm sido desenvolvidos com conteúdos informativos, podendo estar associados ou não a propostas desafiadoras, estimulando 0 aluno a desempenhar um papel mais ativo no entendimento dos conceitos bioquímicos básicos. É neste contexto que se insere este trabalho, constituindo-se num objeto de aprendizagem mediado por computador, no qual se apresenta, interativamente, a "Síntese de Glicogênio Hepático pelas Vias Direta e Indireta". A sua concepção e criação foram realizadas através da plataforma Adobe e seu conteúdo bioquímico foi baseado em informações divulgadas em artigos científicos e em livros texto. Com isto, pretende-se disponibilizar mais um objeto de aprendizagem ao repositório de softwares educacionais, para auxiliar no estudo de Bioquímica, tratando de um tema ainda não contemplado por esta estratégia de ensino.

Palavras-chave: Objeto de aprendizagem; glicogênese hepática; via direta; via indireta

\begin{abstract}
:
Basic Biochemistry subjects usually use lectures or group work dynamics like strategies of theoretical teaching. The review of important concepts is often done through classical memorization exercises in class. On the other hand, in recent years, textbooks have included CDs, which contain several animated figures, associated to hypertexts and linked to websites that enable the construction of meanings of biochemistry knowledge. In addition, learning objects in virtual environments have been developed with informative contents, and may be associated or not to challenging proposals. These could encourage students to play a more active role in their understanding of basic Biochemistry. This work is included in this context and it is a learning object, mediated by computer, which presents interactively the "Synthesis of Hepatic Glycogen by direct and indirect pathways". Its design and creation was performed using the Adobe platform, and its contents were based on biochemical information published in scientific articles and textbooks. The aim is to provide another learning object to the educational software repository which can be useful in the study of a biochemistry topic not yet approached by this teaching strategy.
\end{abstract}

Keywords: Learning object; hepatic glycogenesis; direct pathway; indirect pathway 


\section{INTRODUÇÃO}

Este trabalho apresenta um objeto de aprendizagem (OA) desenvolvido para a área do conhecimento de bioquímica, abordando a "Síntese de Glicogênio pelas Vias Direta e Indireta", como uma proposta alternativa à abordagem tradicional deste assunto em disciplinas de Bioquímica Básica. Nessas disciplinas, normalmente, utilizam-se aulas expositivas, bem como a dinâmica de estudo em grupo, como recurso de ensino teórico e, em alguns cursos, a experimentação laboratorial. Nessa abordagem, não temos de uma forma significativa a utilização de tecnologias digitais de aprendizagem como auxiliares no processo de construção do conhecimento bioquímico, embora os principais livros texto de bioquímica, há mais de uma década, venham acompanhados de CDs contendo várias de suas figuras e representações animadas e associadas a hipertextos e links de páginas da internet, que facilitam a compreensão do conhecimento [1-6].

Assim, como um primeiro movimento de pesquisa, buscamos evidenciar na Revista Brasileira de Ensino e Biologia Molecular (http://www.bdc.ib.unicamp.br/rbebbm) e nos livros texto mais utilizados no meio acadêmico - como o Lehningher [5], o Stryer [7] e o Champe [8] - materiais computacionais que apresentassem, especificamente, o tema da Síntese de Glicogênio pelas Vias Direta e Indireta. Surpreendentemente, verificamos que tal material ainda não havia sido produzido, salvo alguns poucos que não apresentavam um nível de explicação e representação o qual almejávamos [9]. Desta forma, passamos à elaboração do OA aqui apresentado.

Nossa concepção de OAs é aquela que os define por "qualquer entidade, digital ou não digital, que possa ser utilizada, reutilizada ou referenciada durante o aprendizado suportado por tecnologias" [10].

Assim, no processo de elaboração de nosso material, buscamos contemplar a ideia de uma utilização e reutilização e aproximá-lo daquilo que, como tratam Azevedo e colaboradores [11], Galembeck e Torres [12] e Galembeck e colaboradores [13], seja o propósito das tecnologias educacionais: desenvolver propostas desafiadoras e interativas que estimulem o aluno a desempenhar um papel mais ativo na compreensão das etapas envolvidas nos conhecimentos bioquímicos básicos e, mais especificamente, no conhecimento bioquímico proposto por este OA.

Da utilização de OAs podemos destacar, de acordo com Nunes [14], a sua utilidade e capacidade de adaptação às necessidades, habilidades, formação, interesses e estilos cognitivos dos aprendizes envolvidos, além de permitirem alterações no ritmo, dificuldade e outros fatores que envolvem a aprendizagem. Ainda segundo o autor, outra característica, e que por nós é considerada de fundamental importância e como vantagem apresentada pelas tecnologias educacionais, é a que os OAs podem ser acessados de qualquer lugar e a qualquer tempo ${ }^{1}$.

Assim, buscamos na elaboração de nosso material, que pode ser considerado como um Objeto de Aprendizagem Prático conforme Shepherd (apud Bertoletti-De-Marchi [15]), apresentar o processo da síntese do polissacarídeo de reserva animal (glicogênio) no fígado.

Este processo bioquímico é constituído de várias etapas reacionais. Além disso, a glicogênese hepática pode ocorrer em diferentes situações fisiológicas nas quais as vias anabólicas são diferentes: via direta e via indireta. Resumindo, no estado alimentado, a glicose é transportada para o interior do hepatócito pelo GLUT 2, fosforilada à glicose-6P pela glicocinase, isomerizada à glicose-1P pela fosfoglicomutase, transformada em UDPG e doada ao primer de glicogênio, caracterizando a via direta da glicogênese. Por outro lado, após jejum de $20 \mathrm{~h}$, seguido

\footnotetext{
${ }^{1}$ Fator este limitado, obviamente, ao acesso a um computador que disponha dos requisitos mínimos exigidos pelo sowftware empregado. Embora esta restrição pareça muito forte, consideramos que não é determinante, haja vista que $o$ acesso e a disponibilidade de computadores - inclusive as configurações de hardware - cresceram muito nos últimos anos.
} 
de realimentação, a captação direta de glicose pelo fígado nesta situação é considerada pequena e o fluxo gliconeogênico continua ainda por várias horas. A glicose é, então, transformada em intermediários de três carbonos em tecidos extrahepáticos e, após, pela via da gliconeogênese no fígado, os mesmos são convertidos a glicose-6P, a glicose-1P, a UDPG e a glicogênio, caracterizando o processo da via indireta da síntese de glicogênio. A via indireta contribui com 2/3 da síntese total de glicogênio na realimentação [5; 7; 16-18].

Essa complexidade do processo bioquímico de síntese de glicogênio, descrita brevemente, constituiu-se na motivação que nos levou à elaboração de um material mediado por computador que auxiliasse o aluno no entendimento desses processos que requerem a construção de modelos explicativos de elevada exigência de abstração.

\section{MATERIAIS E MÉTODOS}

A evolução das tecnologias disponíveis para a elaboração de materiais educacionais mediados por computador nos permite, hoje, ter uma ampla variedade de softwares que contribuem para sua elaboração. Cada qual com sua peculiaridade, cabendo à equipe de trabalho buscar aquele que melhor se adapte às suas necessidades, tanto relacionadas aos conceitos a serem trabalhados, quanto com o nível de programação exigido. Em nosso projeto, optamos pela utilização de um programa que não exigisse um aprofundamento em linguagens de programação muito elevado, mas que, ainda assim, possibilitasse criar uma liberdade de interação com o usuário. Desta forma, 0 nosso OA foi concebido e desenvolvido através da plataforma Adobe, com a versão 8.0 do programa Adobe Flash. Embora este programa seja de uso comercial e mediante licença ${ }^{2}$, o plug-in necessário para reproduzir o material finalizado pode ser baixado gratuitamente do site da empresa. A vantagem de se optar por essa plataforma é sua universalidade em relação aos sistemas operacionais, sendo compatível com praticamente todos eles.

Além da preocupação com a funcionalidade do objeto, é importante aquela relacionada a um layout agradável e uma navegabilidade fácil e intuitiva, do contrário o aprendiz poderia se cansar ou se desviar da seqüência reacional envolvida, acarretando um prejuízo no aprendizado da síntese de glicogênio.

Outro fator importante para o desenvolvimento do OA aqui apresentado é que sua utilização visa ser uma forma de complemento/apoio a uma aula presencial. Assim, alguns aspectos importantes ao longo do processo sintético devem ser intermediados pelo professor. Pensamos que dessa maneira o objeto possa ser configurado como um facilitador da aprendizagem e auxiliar no processo de ensino do professor das disciplinas de Bioquímica Básica.

A partir dessas considerações, construímos o OA. Em sua elaboração, optamos por utilizar um arquivo principal que, quando solicitado, realiza um call (chamado) nos arquivos complementares. Esta composição pode ser melhor entendida através da Figura 1. Este tipo de configuração do OA foi escolhido por que facilita no processo de re-edição do mesmo, visto que, tão logo forem realizados os processos avaliativos do material, modificações provavelmente terão de ser realizadas, com o intuito de tornálo cada vez mais próximo às necessidades daquele que o utiliza. Esta estrutura possibilita também que o usuário realize uma navegação livre, podendo acessar um conteúdo especificamente (fora da ordem) ou seguindo uma proposição seqüencial apresentada no Menu de Tópicos. Estas e outras características serão apresentadas a seguir.

\footnotetext{
${ }^{2}$ Ou seja, deve ser comprada a licença para sua utilização.
} 


\section{RESULTADOS E DISCUSSÃO}

O objeto tem um design diferenciado, pois, embora sua concepção tenha sido modelada a partir das páginas de internet e de outros softwares do segmento da área de conhecimento da bioquímica, buscamos em suas linhas curvas, posicionamento de botões e forma de navegação dar uma característica mais fluida e agradável ao conteúdo nele expresso.

No momento em que é acessado, tem-se de forma dinâmica algumas informações a respeito da origem institucional dos autores. Em seguida, o usuário é levado à tela inicial (Figura 2), onde são apresentados ícones que o guiam durante a navegação.

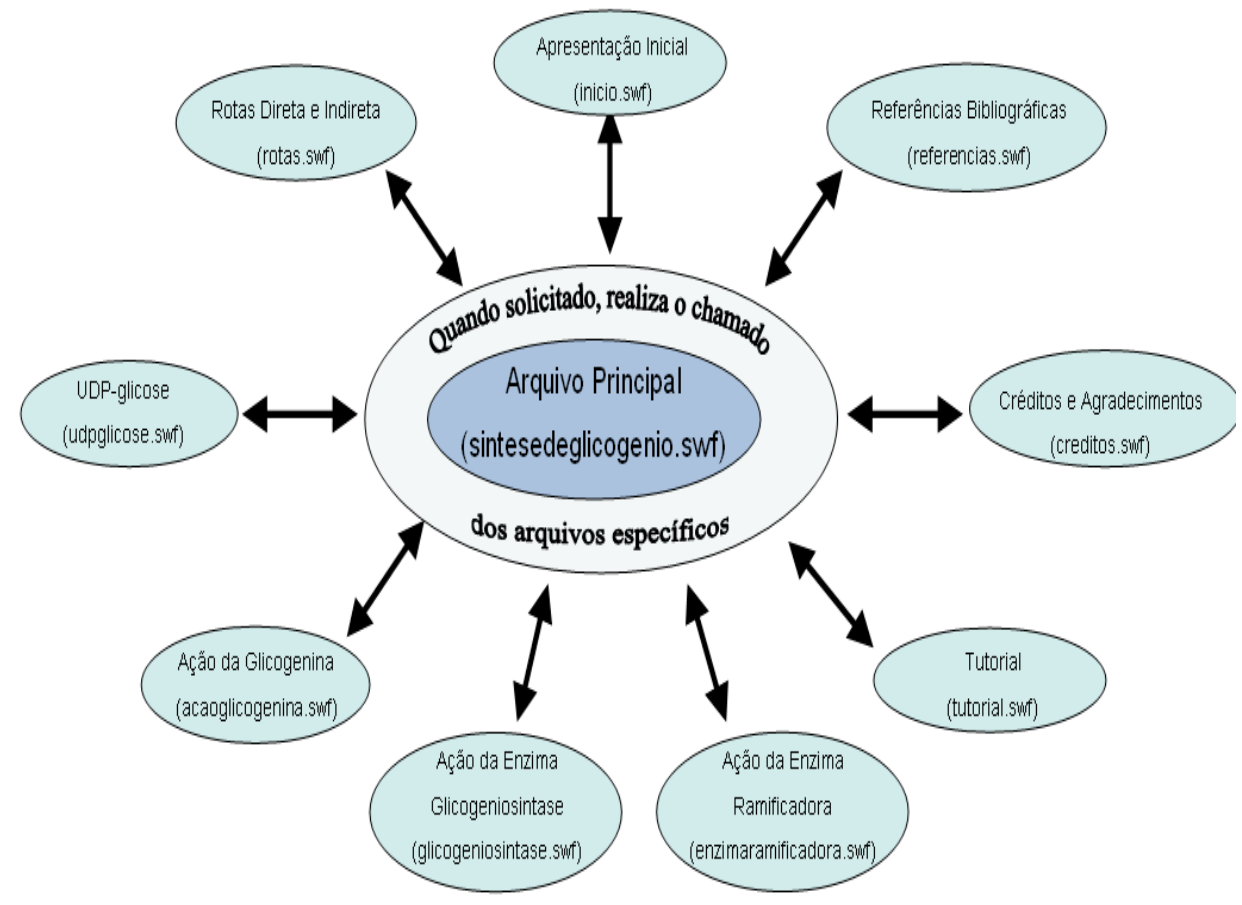

Figura 1: Apresentação da árvore de arquivos utilizados no OA desenvolvido.

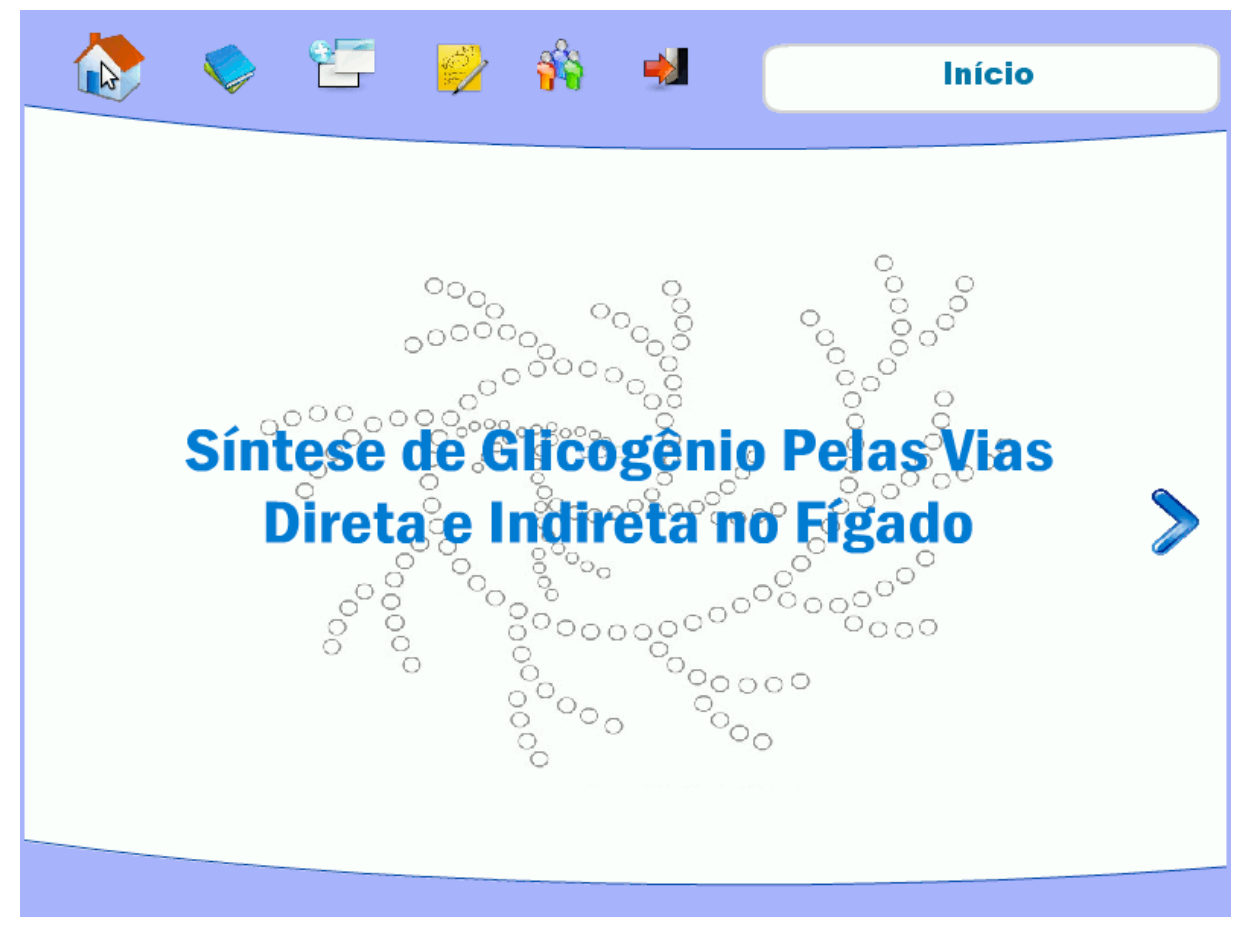

Figura 2: Tela inicial do Objeto de Aprendizagem. 
Nas Figuras 2 e 3 é possível observar, em sua parte superior, os botões do menu principal, os quais são, da esquerda para a direita: Início, Tutorial, Menu de Tópicos, Referências (bibliográficas), Créditos e Sair do Programa. Ao lado deles, pode-se observar um quadro branco, onde, ao passar o mouse sobre esses botões, pode-se visualizar seus nomes. Ao clicar no botão Menu de Tópicos, abre-se uma guia que contém os tópicos/etapas relacionadas ao processo de síntese do glicogênio (Figura 3). Nessa figura, observa-se que existem cinco tópicos, a saber: 1) UDP-glicose; 2) Glicogenina; 3) Glicogênio Sintase; 4) Enzima Ramificadora; 5) Rotas Direta e Indireta. Nota-se, da comparação entre as Figuras 2 e 3, que, antes de clicar sobre o botão Menu de Tópicos, este possui um sinal azul com um símbolo (+). Quando ele é clicado, passa a apresentar um sinal vermelho com outro símbolo (-). Esta simbologia busca dar ao OA uma dinâmica mais intuitiva durante sua utilização. Pensando na funcionalidade do programa, o usuário poderá navegar por todo o conteúdo utilizando-se apenas dos botões referidos.

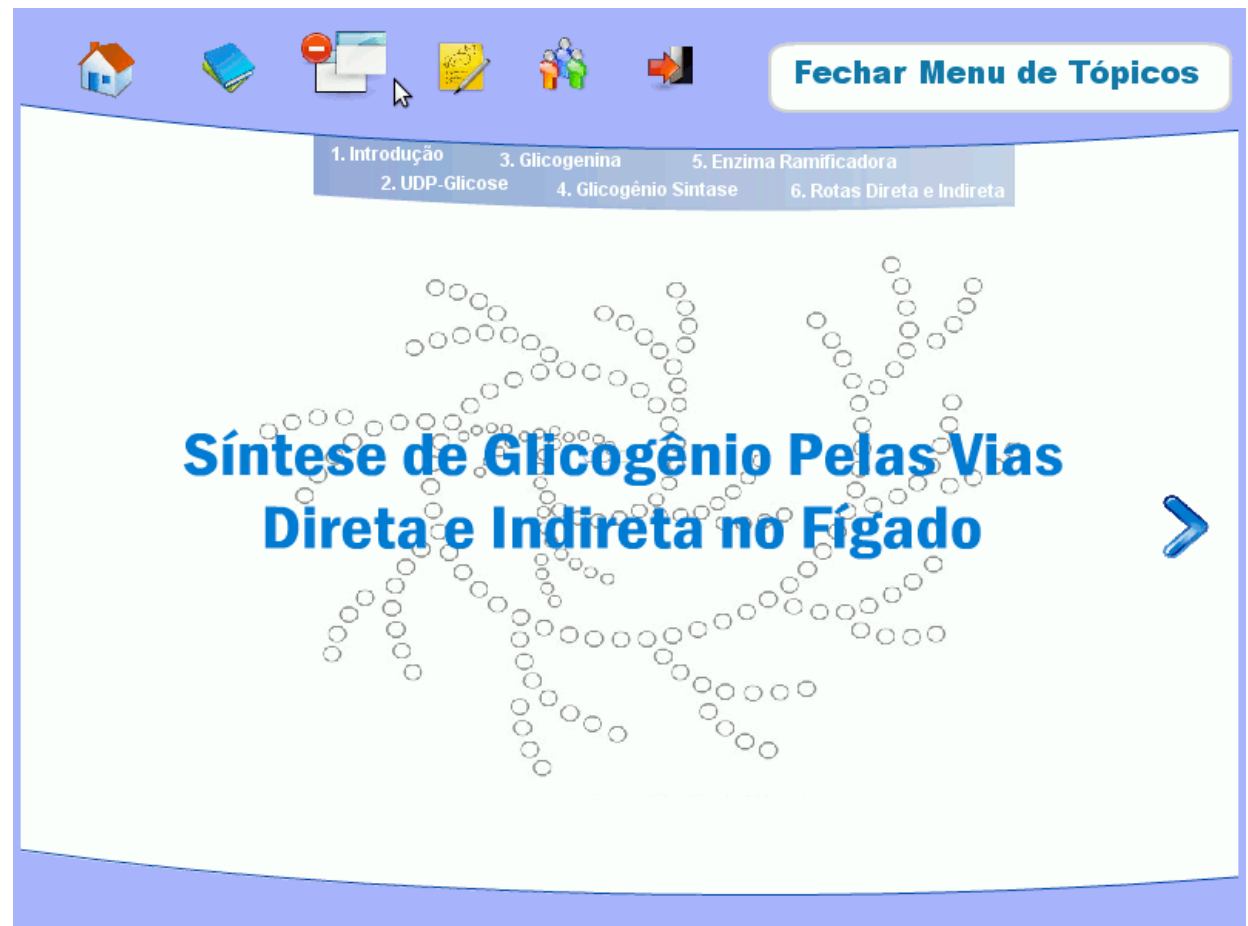

Figura 3: Detalhe do Menu de Tópicos

De maneira complementar e para facilitar o entendimento da seqüência de processos envolvidos na síntese bioquímica do glicogênio, em cada etapa existe uma seta (>) que, ao passar o mouse sobre ela, indica qual o próximo tópico (dentre os cinco citados). Um exemplo desta funcionalidade pode ser observado na Figura 4. Outra funcionalidade que disponibilizamos neste $O A$, e que visa facilitar a compreensão da relação da representação macroscópica com a microscópica dos processos bioquímicos, é a utilização de imagens/figuras inseridas na parte inferior da tela, que podem ser ampliadas, como na Figura 5. 


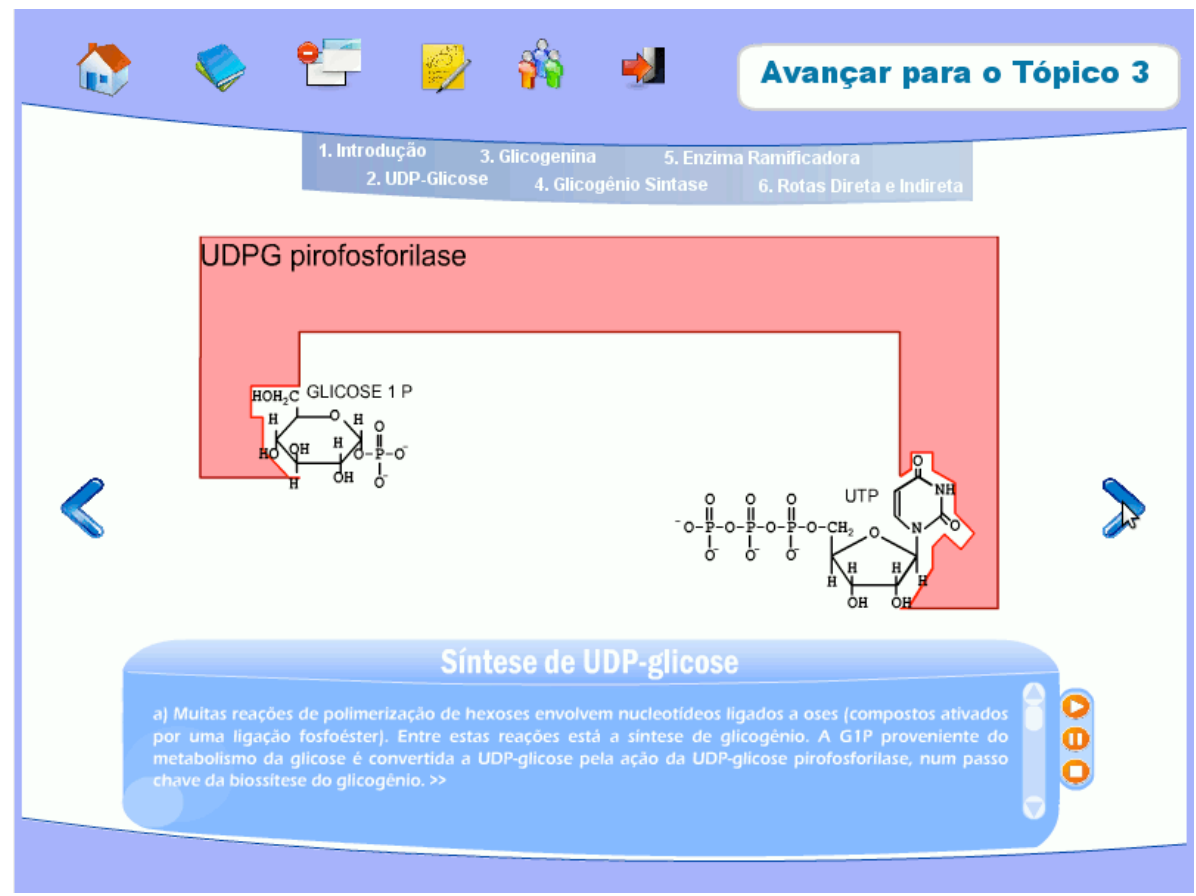

Figura 4: Utilização da seta azul à direita para navegar ao próximo tópico.
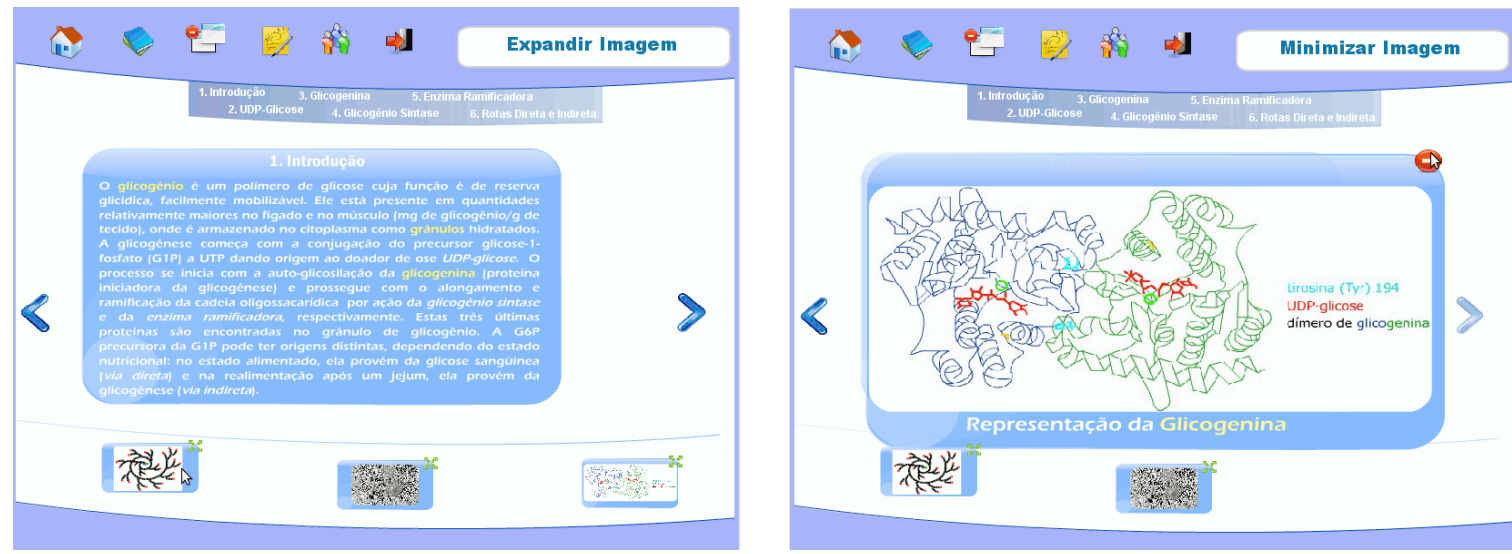

Figura 5: Capturas de duas telas - a primeira, antes de ampliar a imagem localizada no canto inferior direito, e a segunda, após a ampliação.

Como citado, a preocupação de tornar o OA intuitivo, simples, na sua utilização levou-nos a utilizar uma uniformidade de navegação pelos tópicos. Desta forma, como podemos visualizar na Figura 6, os cinco tópicos abordados possuem: um espaço destinado à informação conceitual; e outro destinado à representação do processo bioquímico na forma de animação. Este último consideramos ser o diferencial deste $O A$, pois auxilia no processo de abstração necessário à compreensão das reações químicas constituintes da rota metabólica em estudo, diferentemente de imagens e informações estáticas usualmente apresentadas.

Embora as representações utilizadas possuam um caráter simplificador do processo envolvido, optamos, mesmo assim, por utilizá-las, pois permitem priorizar os aspectos mais relevantes do processo de síntese do glicogênio animal no fígado. 


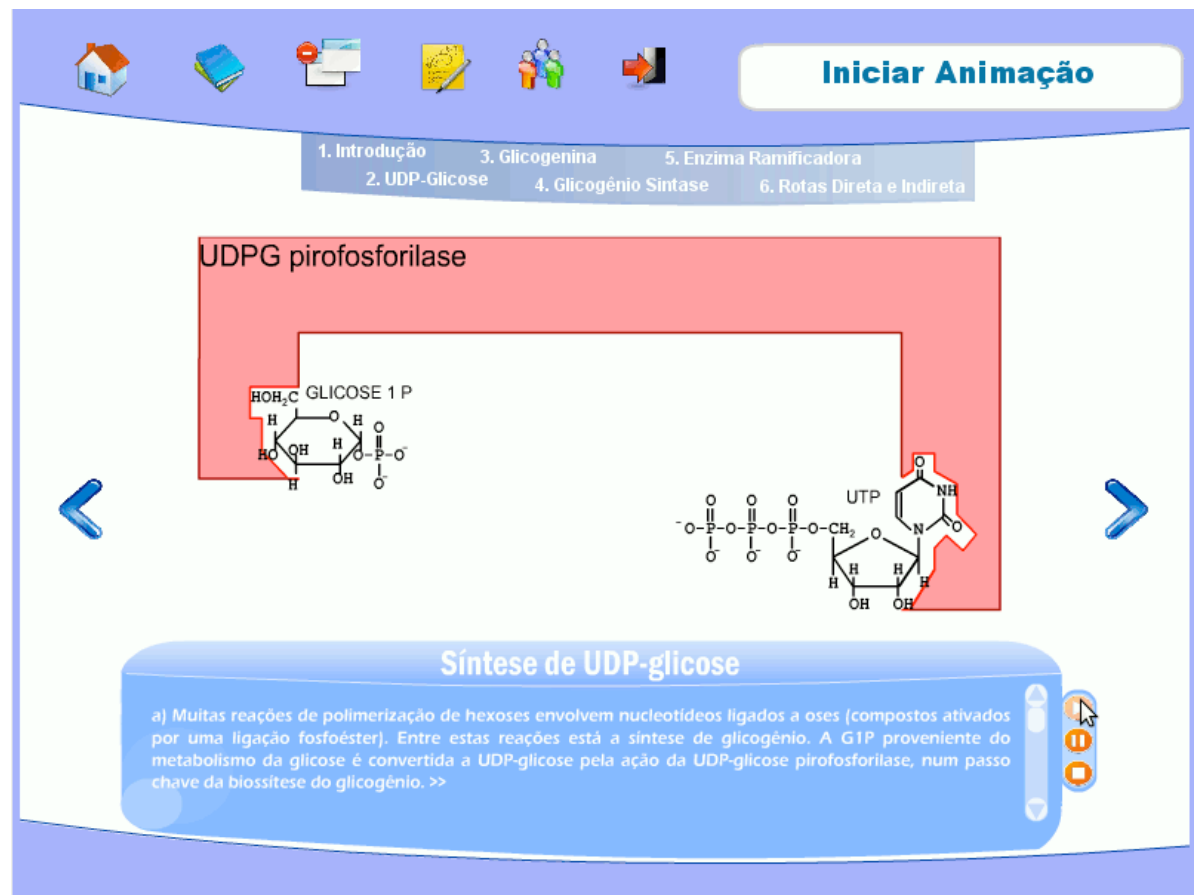

Figura 6: Apresentação do tópico 2 (UDP-glicose). Acima, animação do processo bioquímico. Abaixo, informações conceituais do processo representado na animação.

Para seguir na leitura e visualização da animação referente ao tópico, basta que o usuário clique na barra de rolagem ao lado do texto. Esta forma de apresentação dos tópicos permite ao usuário, uma vez entendido o funcionamento de um deles, navegar facilmente pelos outros.

\section{CONCLUSÃo}

Este OA foi concebido com intuito de facilitar a compreensão do processo de glicogênese através da interatividade com o usuário. A proposição da utilização de representações na forma de animações e figuras, seguidas de textos explicativos, busca facilitar e auxiliar no entendimento dos processos e reações químicas que ocorrem na Síntese do Glicogênio - dividido neste OA na forma de tópicos, os quais podem ser vistos ordenadamente, seguindo a seqüência de etapas envolvidas na síntese do glicogênio, ou de forma aleatória. Essa característica é dada em função dos tipos de navegação pensados possíveis pelo usuário e da programação escolhida.

Pensamos que o material proposto não se configura como uma proposta de navegação autônoma, devido à complexidade do processo representado, sendo recomendado seu emprego mediante a orientação de um professor.

No momento, o OA em questão encontra-se em fase de apresentação aos alunos de graduação e de pós-graduação, assim como, aos professores do Departamento de Bioquímica da Universidade Federal do Rio Grande do Sul - Brasil. Embora ainda pequeno o número de validações, que por ora nos impede de uma análise estatística, vemos nas avaliações preliminares resultados positivos, acompanhadas de palavras de incentivo para a continuidade na criação de outros objetos de aprendizagem que abordem distintos mecanismos de controle do metabolismo intermediário, mecanismos estes específicos do campo de conhecimento da bioquímica.

Na projeção de ações futuras, pretendemos disponibilizar à comunidade científica o objeto aqui apresentado, através da submissão do mesmo a sites específicos ao campo da bioquímica, como o da Revista Brasileira de Ensino em Bioquímica e Biologia Molecular, com a possibilidade de acesso no endereço 
http://www.bdc.ib.unicamp.br/rbebbm. Além disso, melhoraremos o processo de avaliação do software, buscando, deste modo, aprimorá-lo, uma vez que é nessa possibilidade de reutilização e aprimoramento que reside um dos aspectos de relevância dos objetos de aprendizagem. No momento, é possível acessar o objeto em questão no endereço http://www.iq.ufrgs.br/aeq/softwares/index.php, referente ao grupo de pesquisa na Área de Educação em Química, do Instituto de Química, da Universidade Federal do Rio Grande do Sul.

\section{AGRADECIMENTOS}

Ao Prof Dr Marcos Luiz dos Santos Perry (in memoriam) pelos constantes ensinamentos enquanto estava entre nós no Departamento de Bioquímica-UFRGS. Ao PPG-CB:Bioquímica e ao Prf. Dr Clóvis W.D. Wannmacher por permitir o desenvolvimento deste projeto junto à disciplina de Práticas de Ensino pela aluna Luciene P. Vianna. CESUP (Centro Nacional de Supercomputação - UFRGS) pelo incentivo da jornalista Maria Isabel Timm (in memoriam) CINTED (Centro Interdisciplinar de Novas Tecnologias na Educação) pela oportunidade da co-autora Luciene Pinheiro Vianna participar do curso "Projeto e Desenvolvimento de Materiais Educacionais com Flash 8 - 10a. Edição". SEAD (Secretaria de Ensino à Distância UFRGS) pelo apoio ao grupo.

\section{REFERÊNCIAS BIBLIOGRÁFICAS}

[1] T. M. DEVLIN (1998) Textbook of Biochemistry with Clinical Correlations, 5rd ed., Academic Press, New York.

[2] D. VOET, J. G. VOET, C. PRATT (1999) Fundamentals of Biochemistry, John Wiley \& Sons, Inc, New York.

[3] D. L. NELSON, M. M. COX (2000) Lehninger -Principles of Biochemistry, 3rd ed., Worth Publishers, New York.

[4] (2004) Lehninger - Principles of Biochemistry, 4rd ed., W. H. Freeman and Company, New York.

[5] (2008) Lehninger - Principles of Biochemistry, 5rd ed. W. H. Freeman and Company, New York.

[6] A. MARZZOCO, B. B. TORRES (2007) Bioquímica Básica, $3^{a}$ ed., GuanabaraKoogan, Rio de Janeiro.

[7] J. M. BERG, J. L.TYMOCZKO, L. STRYER (2002) Biochemistry, 5rd ed., W. H. Freeman and Company, New York.

[8] P. CHAMPE, R. A. HARVEY, D. R. FERRIER (2009) Bioquímica Ilustrada, Tradução Carla Dalmaz et al. 4rd ed., Artmed, Porto Alegre.

[9] M. A. Wells; J. Tupy (1998) An electronic companion to Biochemistry, Cogito learning media, Inc, San Francisco.

[10] IEEE. Learning Object Metadata (LOM) Working Group 12. Institute of Electrical and Electronics Engineers, Inc. Disponível em: <http://Itsc.ieee.org/> Consultada em 05 outubro de 2009. 
[11] A. M. P. AZEVEDO, G. LAZZAROTTO, M. I. TIMM, M. A. ZARO (2004) Relato de uma experiência com o uso do Diagrama Metabólico Dinâmico Virtual do Ciclo de Krebs, RENOTE. Revista Novas Tecnologias na Educação, Disponível em: <http://www.cinted.ufrgs.br/renote/mar2004/artigos/07-

elatodeuma_experiencia.pdf>. Consultada em 07 de outubro de 2009.

[12] E. GALEMBECK, B. B. TORRES (2007) CTE (Cadeia de Transporte de Elétrons), Revista Brasileira de Ensino de Bioquímica e Biologia Molecular, 30 jul. 2007. Disponível em: <http://www.ib.unicamp.br/Ite/rbebbm/visualizarMaterial. php?idMaterial=524>. Consultada em 12 de outubro de 2009.

[13] E. GALEMBECK, C. E. S. P. FILHO, B. B. TORRES (2007) A cinética da reação enzimática, Revista Brasileira de Ensino de Bioquímica e Biologia Molecular, 03 sep. 2007. Disponível em: <http://www.ib.unicamp.br/lte/rbebbm/visualizarMaterial.php?idMaterial=527>. Consultada em 12 de outubro de 2009.

[14] C. A. A. NUNES (2002) Criação, produção e uso de Objetos de Aprendizagem. In IX Congresso Internacional de Educação a Distância, São Paulo, SP.

[15] A. C. BERTOLETTI-DE-MARCHI, F. B. SILVA (2004) O Desenvolvimento de Objetos de Aprendizagem no Museu Zoobotânico Augusto Ruschi. RENOTE. Revista Novas Tecnologias na Educação Vol. 2, No. 2.

[16] C. KUNST, R. KLIEGMAN, C. TRINDADE (1989) The glucose-galactose paradox in neonatal murine hepatic glicogen synthesis, Am. J. Physiol. 257 (Endocrinology and Metabolism) 20: E697-E703.

[17] D. TOSH, G. BERESFORD, L. AGIUS (1994) Glycogen synthesis from glucose by direct and indirect pathways in hepatoyte cultures from different nutritional states. Biochem. Biophy. Acta, 1224, 205-212.

[18] B. J. GIBBONS, P. J. ROACH, T. D. HURLEY (2002) Crystal structure of the autocatalytic initiator of glycogen biosynthesis, glycogenin, J. Mol. Biol. 319, 463-477. 